\title{
Psychological Distress of Immigrant Population in Host School Systems and the Critical Period for Second Language Development: A Review
}

Sandra Figueiredo ${ }^{1 *}$, Margarida Alves Martins ${ }^{2}$ and Carlos Fernandes da Silva ${ }^{3}$

${ }^{1}$ Autonomous University of Lisbon Luís de Camões, Lisbon, Portugal

${ }^{2}$ University Institute, Lisbon, Portugal

${ }^{3}$ Department of Education and Psychology, University of Aveiro, Aveiro, Portugal

\begin{abstract}
Immigrant students attending schools in the host country have different cognitive, cultural and linguistic backgrounds that might influence their inclusion process. This process is enhanced by success attained in academic context and by psychological adaptation. Academic difficulties and limited proficiency could motivate psychological distress and anxiety levels that constraint development and learning of newer immigrants' generation. Maladaptive behaviours might occur with different severity among non-native school children attending to their ethnic differences, cultural traits, length of residence and age. Also the maturational characteristics that interfere with the achievement of native children are probably different from the cognitive maturation that explain the performance of immigrant students, in the same schooling years. In this critical review two main concerns are explored as research based evidence and also as theoretical hypotheses: how distinct immigrant groups differ in psychological distress according to pre-migration and post-migration experiences, and how immigration may change the cognitive processing abilities and the brain development.
\end{abstract}

Keywords: Psychological distress; Immigrants; Ethnic differences; School achievement; Critical period

\section{Introduction}

Academic issues and mental health disturbance (mainly depression symptoms) are recently being studied as correlated in immigrant young populations based on their use of counselling services [1]. Associated with academic limited skills, there are mood fluctuation and psychotic disorders evolving and depending on the post-migration effects [2]. Different racial and ethnic groups suffer from psychological distress of distinct severity and have different educational conflicts [1]. Academic distress is the main cause leading immigrant student populations to counselling services for help with adaptation, attainment of higher educational grades and well-being at school and university $[3,4]$. Very recent research has found that Asian American students experience higher levels of stress and anxiety, followed by Hispanic immigrants, contrary to other racial groups [5].

Psychological distress symptoms can also take (or be masked in) different forms according to culture, which means that evaluation studies of these populations may be insufficient. On the other hand, Asian Americans and Hispanic Americans resort to psychological services less than other ethnic peers, which may explain fewer studies about the distress and unadapted behaviour of immigrant groups other than Asian or Hispanic [5]. Attending to this evidence, is of upmost importance to examine how these immigrant minorities are growing in the traditional and in the new receiving countries and how their trends are changing. Studies of Chow et al. [6] and of Takeuchi et al. [7] illustrate the immigration trends, in specific different periods (study 1[6]: 1995 and study 2[7]: 2002/2003), and matching the mentioned groups above, considering demographics, the health services attendance (problems observed and quality of services mainly correlated to the poverty areas) and the most representative psychological disorders determined by immigrant groups, but only for the US context. Asian and Hispanic, for that context, are the populations with higher risk for psychological disorders. The previous literature only refers to the North America as the main host country of immigration, further research should examine the European and also the Asian contexts as host settings, mainly for the school population that is our most concern to study.

For European settings there are less studies to determine the attendance of immigrants for mental health services, despite of the use of other type of health services (not necessarily related to psychological disorders). For the European countries, we observed a multicountry study [8] with a variety of percentages for health problems and limited health perception of immigrants in several European countries, considering the 2004-2005 period only. We had perceived that the research studies and the research scholars for this matter in immigration among European countries are still scarce and focused the adult populations, reporting to a systematic review that had considered the period 2013-2016 [9].

Populations under age 18 are the less examined for the previous context of research, managing their own school processes and the early educational development. The minority groups are specifically identified in schools as being at risk with regard to educational milestones [10,11]. Hispanic and mainly the Asian population, which are populations with great diversity considering their several countries of origin, have low scores in proficiency tests and difficulty in engaging in host school contexts $[1,12,13]$. Here we may advance a "match" hypothesis that correlates the mental health problems observed for these two immigrant groups as adults and the academic limitations of the same groups as young students.

Regarding the proficiency and difficulties, research with minorities

*Corresponding author: Sandra Figueiredo, Post-doctoral researcher Autonomous University of Lisbon Luís de Camões, Lisbon, Portugal, Tel: +00351218811700; E-mail: sandradfigueiredo@ua.pt

Received: April 20, 2018; Accepted: May 05, 2018; Published: May 12, 2018

Citation: Figueiredo S, Martins MA, da Silva CF (2018) Psychological Distress of Immigrant Population in Host School Systems and the Critical Period for Second Language Development: A Review. Clin Exp Psychol 4: 192. doi: 10.4172/24712701.1000192

Copyright: (c) 2018 Figueiredo S, et al. This is an open-access article distributed under the terms of the Creative Commons Attribution License, which permits unrestricted use, distribution, and reproduction in any medium, provided the original author and source are credited. 
requires clarifying the ethnic and minority concepts (strongly associated to "immigrant" and "racial" terminology in the literature) as well as the variables related to learning styles and proficiency levels. In our research we prefer to adopt the concepts 'immigrants' and 'minority' as being related but we are aware of the groups' differences considering their ethnic and linguistic characteristics [14]. Concerning the learning styles of those groups, there is no examination of the typology of stimuli applied to the language and academic tests that could affect the proficiency diagnostic and also mask these students' academic skills $[15,16]$. Table 1 presents a dataset from a study Figueiredo et al. [13] about the performance differences of six groups ( $\mathrm{N}$ ranging from 67 and 101 subjects) of immigration in Portugal (under age 18). The lowest scores were found for Asian immigrants (countries of origin such as Blangladesh), except for the tasks related with recall and unfamiliar sounds listening.

Furthermore, the correlation between ethnic preferences for second language (L2) learning and for responding to language and verbal reasoning tasks has not been clearly researched in the fields of

\begin{tabular}{|c|c|c|c|c|}
\hline \multicolumn{2}{|c|}{ Tasks (Factors) } & $\mathbf{N}$ & Mean & S.D \\
\hline \multirow{7}{*}{$\begin{array}{l}\text { Verbal reasoning, } \\
\text { writing, léxicon }\end{array}$} & China & 16 & 62,63 & 23,301 \\
\hline & South America & 4 & 84,50 & 17,972 \\
\hline & East Europe & 16 & 73,81 & 15,673 \\
\hline & Africa & 13 & 67,77 & 15,412 \\
\hline & Western Europe & 9 & 79,22 & 20,795 \\
\hline & Asia & 9 & 63,67 & 24,290 \\
\hline & Total & 67 & 69,97 & 20,212 \\
\hline \multirow{7}{*}{ Recall } & China & 15 & 10,20 & 5,532 \\
\hline & South America & 5 & 17,40 & 5,459 \\
\hline & East Europe & 24 & 14,42 & 9,758 \\
\hline & Africa & 16 & 14,88 & 5,620 \\
\hline & Western Europe & 10 & 16,60 & 7,806 \\
\hline & Asia & 11 & 16,64 & 14,637 \\
\hline & Total & 81 & 14,48 & 8,887 \\
\hline \multirow{7}{*}{ Oral decoding } & China & 22 & 6,00 & 1,512 \\
\hline & South America & 5 & 7,00 & 1,000 \\
\hline & East Europe & 25 & 5,84 & 1,748 \\
\hline & Africa & 15 & 6,60 & 1,682 \\
\hline & Western Europe & 10 & 6,70 & 1,636 \\
\hline & Asia & 11 & 5,82 & 2,316 \\
\hline & Total & 88 & 6,17 & 1,717 \\
\hline \multirow{7}{*}{$\begin{array}{l}\text { Phonological } \\
\text { decoding }\end{array}$} & China & 24 & 1,92 & 1,139 \\
\hline & South America & 6 & 2,50 & 1,225 \\
\hline & East Europe & 28 & 2,43 & 1,136 \\
\hline & Africa & 18 & 1,61 & 1,037 \\
\hline & Western Europe & 11 & 2,00 & 1,183 \\
\hline & Asia & 14 & 1,50 & 1,019 \\
\hline & Total & 101 & 1,99 & 1,145 \\
\hline \multirow{7}{*}{$\begin{array}{l}\text { Unfamiliar sounds/ } \\
\text { listening }\end{array}$} & China & 22 & 3,3182 & 1,21052 \\
\hline & South America & 5 & 4,0000 & 1,00000 \\
\hline & East Europe & 20 & 3,4500 & 1,14593 \\
\hline & Africa & 15 & 3,9333 & 1,94447 \\
\hline & Western Europe & 10 & 3,0000 & 1,63299 \\
\hline & Asia & 12 & 3,7500 & 1,42223 \\
\hline & Total & 84 & 3,5238 & 1,41826 \\
\hline
\end{tabular}

Note: $\mathrm{SD}=$ Standard Deviation, Posthoc=Tukey Alpha (0.05) tests were used to ascertain the significant statistical differences observed among groups.

Table 1: Performance variability among six immigrants' groups in Portugal: Asian immigrants' disadvantage at schools (period 2013-2016).
Psychology and Education. On the other hand, Neuropsychology has contributed to the understanding of the cognitive mapping of different speakers in several languages as L2 and specifically to the understanding of difficulties and delays on auditory processing $[17,18]$. However, the English as L2 is the main known context for the analysis of performance (and difficulties) differences of immigrant young students. Individuals react differently to input and stimuli during task processing due to their prior cognitive strategies developed and mapped in their home language (L1) and they are constrained by prior schooling in their countries of origin [19]. Additionally, students might be facing different constraints when they are processing in distinct (and distant) languages other than English.

Studies in Clinical and Cognitive Psychology areas still fall with the research to examine how immigrants differ across minority groups with regard to their performance and to their (academic related) psychological distress symptoms $[20,21]$. The research of mental health of immigrant populations is a gap mainly for European settings [20,22] where immigrant and refugees are increasing in number and also in diversity respecting countries of origin. European studies, especially those conducted in Norway [20], had developed interesting data on the risk factors and on the mental health predictors that we are not completely aware concerning our immigrant populations. However, these studies remain focused in adult population, not related to the childhood and immigrants' performance at school.

In this review, performance refers to the (academic) achievement at the school level and to the cognitive strategies that different young groups may apply for the target learning in the countries of destination. For adults, the performance concerns labour market and language learning which implies other scenarios that also should be further explored. Psychological distress may explain the academic failure and maladaptive behaviours during childhood and with consequences for the adult life. It is crucial to understand how children are responding to host institutions and how they perceive their own language and academic difficulties. Then, how to develop valid measures and intervention, focusing the second generation of immigrants and their mental health development [20].

According to the previous literature, the academic failure and the associated psychological distress of specific immigrant' groups are cognitively related [23]: cognitive mapping and consolidated strategies to respond to specific tests and stimuli. The cognitive mapping is intrinsically related to the home language and to prior knowledge (their educational paths in their home schools) that immigrant and ethnic groups have in different ways. There is research on the stimuli and on the linguistic level of difficulty applied to other areas such as mathematic evaluation tests at school [24]. Results from that research showed that linguistic minorities (immigrants) struggle more significantly than their native peers when the maths tests are linguistically difficult. The difficulty was described as lexical and syntactical properties that appear in the tests requiring high level of understanding in terms of academic language competency. It is more likely that immigrant young students find it hard to decode formal language items (in second language) such as passive voice, formal lexicon, and long and complex sentences. Table 2 established, based in causal relationships through confirmatory factorial analysis, how the young immigrant students (in Portuguese context) could be influenced in performance by schools, especially considering the different schools and their resources (among them we include the teachers' styles). Resources included also the proficiency testing procedures [25].

In the school variable we considered the proficiency tests as 


\begin{tabular}{|c|c|c|c|c|c|c|}
\hline \multicolumn{2}{|c|}{ School variable } & Recall & Picture naming & Similarities & Morphology & Cognates \\
\hline \multirow{3}{*}{ School 1} & Mean & 4,75 & 33,4444 & 6,0000 & 4,2000 & 3,0000 \\
\hline & $\mathrm{N}$ & 8 & 9 & 10 & 10 & 10 \\
\hline & SD & 1,982 & 4,95255 & 3,01846 & 2,09762 & 1,63299 \\
\hline \multirow{3}{*}{ School 2} & Mean & 3,40 & 22,3333 & 2,3333 & 1,5000 & 1,7143 \\
\hline & $\mathrm{N}$ & 5 & 6 & 6 & 6 & 7 \\
\hline & SD & 1,673 & 15,27962 & 3,38625 & 1,51658 & 1,60357 \\
\hline \multirow{3}{*}{ School 3} & Mean & 4,46 & 32,2143 & 7,3571 & 5,0000 & 3,9286 \\
\hline & $\mathrm{N}$ & 13 & 14 & 14 & 14 & 14 \\
\hline & SD & 2,634 & 6,39926 & 3,47756 & 2,41788 & 1,20667 \\
\hline \multirow{3}{*}{ School 4} & Mean & 4,00 & 29,0769 & 5,3077 & 3,0909 & 3,0833 \\
\hline & $\mathrm{N}$ & 12 & 13 & 13 & 11 & 12 \\
\hline & SD & 3,303 & 11,13898 & 4,11065 & 2,58668 & 1,83196 \\
\hline
\end{tabular}

Table 2: Performance scores in different tasks answered by immigrant children immersed in four selected schools in lisbon district, Portugal (period 2013-2016).

resources as a main covariate [25]. However, there is no sufficient knowledge about the typology of stimuli in the tests, especially in L2 proficiency testing. Research on images as visual stimuli for specific tasks demonstrates that pictures with cultural meaning can disrupt the decoding process during a task and with emotional blocking effect [26]. In other analyses, despite the importance attributed to the listening inputs during evaluation tests for immigrant students [27], the auditory discrimination, mainly through listening comprehension tasks, can be highly difficult at the early stages [28] and also less effective for L2 learners considering a long-term exposure (towards auditory stimuli and less visual and orthographic stimuli). Those difficulties may become perceived by students as an 'expired' learning opportunity that will likely result in high anxiety and low self-esteem with negative impact on their academic goals or school dropout.The 'exposure' is a wellknown concept in the studies of immigrants' cognitive development and in the second language research and should be noticed also for the understanding of reactions and emotional states that immigration' groups exhibit when they undertake tests, language or psychological tests, in the countries of destination. Recent findings [1] have noted that Asian young immigrants showed higher anxiety levels compared with the other immigrant groups, after the same anxiety tests had been administered (to the other groups). Other data [29] revealed that the Asian group was a high-risk population after immigrating to the US and receiving psychological aid for severe high levels of stress, and that factor correlated to their academic results and expectations.

Another perspective, in Psychology, should examine the hypothesis that the same minorities, as they were previously identified, may experience different difficulties and anxiety when they are established in other contexts such as the European, differently from the American settings [30]. Considering European receiving countries, still it is important to consider significant well-being differences among natives and immigrants (with evidence of high rate of psychological needs for the second group) as well as the specificities of mental health of each European population, their native and immigrant [31]. The mobility (pre-migration) that motivates immigration (such as the case of refugees) and the culture specific traits of each minority determine the emotional problems and the coping behaviours with more or less implication for the academic attainment [32]. The main moderator may be identified in the school strategies and counselling services that the host country develops for their immigrants and refugees. Still, there is no sufficient research about the reaction' differences of minority' groups to school (and psychological) tests, and also little is known about the learning processes observed in the same cultural groups but living in different host countries.

In a study Leveles and Dronkers [33] conducted to evaluated the pre-migration causes (factors and episodes that motivate the emigration) and the post-migration effects (adaptation factors and episodes after entrance in the country of destination) on the well-being of immigrants, the main results proved to be more statistically significant for the post-migration effects as a variable to explain distress. Again, the Asian group might experience the post-migration effect more than the pre-migration causes, and this had impact on their grades. These data highlight the reality of refugees, who are one of the main groups suffering from pre-migration effects [34,35]. Still, few studies examine the post-migration effects on refugees. In previous studies about immigrants in the North and South of Portugal [36], the younger Asian Portuguese students also showed high levels of academic failure and additional cognitive disproportion in language and verbal reasoning tasks compared with the other groups of immigrants in the same schools. In Portugal, as likely in other countries, the Asian immigrants can be divided into two main groups: China and Asian Indian (main countries of origin: Bangladesh, Nepal and India). Normally the second group has the lowest achievement in our evaluation studies contrary to the Chinese students with better scores, which is different, for example, from the grades obtained for similar samples in a study previously carried out [1].

Considering that evidence suggests that post-migration is more difficult for specific non-native individuals or groups compared to others, it is important to carry out research that examines different minorities, especially children to assess their proficiency, academic and psychological need at their school entry. In sum, evidence shows that second generation of immigrants attending schools in the countries of destination resort to psychological counselling services due to problems with distinct levels of severity, mainly reporting academic distress and failure that cause well-being disturbances [1,5,29]. These disturbances are different depending on the ethnic groups and on their academic complaints, which are mostly related to language proficiency [1] attending that American Psychological Association [14] confirmed the existence of guidelines according to specific ethnic minority groups with regard to their psychological assessment, it should be coherent that the academic and language evaluation might also require a norm for specific immigrant minorities, and distinguish them from other young non-native learners such as the refugees [34].

\section{New Neural Systems and Evolution Caused by Immigration}

Along with the psychological distress related to the immigrant population specificities, the mobility worldwide has other implications of interest for Psychology, especially for the understanding of learning processes and neural systems evolution. After the first studies that 
confirmed that critical period diminishes its opportunity window for languages acquisition (and for second language acquisition) around the puberty phase $[37,38]$, newer researches proved that the optimal period could be extended during the adolescence and authors revealed support to consider the adult age [39,40]. Also evidence highlighted cross-linguistic factors that are involved to produce differences for critical period robustness [41,42]. That robustness, however, could be reduced and compromised by the levels of eventual distress caused by events of disruption at school that immigrants may experience.

Distress is expected to be more pronounced in immigrant adolescents than in children maintaining that past results determined adolescents as potentially developing more emotional blocking toward the second language learning $[43,44]$ and more negative attitudes toward the host country and their inclusion strategies (such as the availability of mental health services for immigrants), mainly felt by immigrant parents [45]. These circumstances would interfere, and controlling for ethnic group differences, with the 'optimal' conditions of psychological adaptation [46].

Studies also supported that the sensitive periods should be analyzed in a diversity of timings according to the several levels (syntactic, semantics, lexicon, grammar, prosody) of language maturation $[37,47]$. Despite of the divergence between authors that struggle to determine a "deadline" for the critical period, we understand that in the last four decades there was an advance of the age indicator. Moreover, in the more recent studies we have evidence of children reaching easier more languages at the same time (becoming bilingual), and adults being more proficient in a second language; on the other hand, concerning clinical implications for cognitive development, we know that a bilingual brain delays the dementia diseases [48]. We advance as hypothesis that both high cognitive functioning promoted by bilingualism or multilingualism and the extended critical period are indicators that human brain is evolving and memorizing new survival strategies. Further studies should examine how those strategies relate to the psychological distress and anxiety states in the post-migration period.

All these conditions, that are not only age dependent, could be conducting to a new evidence that affects the critical period delimitation, mainly for the Psychology field. We consider advancing age as a fact to explain the low consensus on the optimal period for language learning is an evidence of a new cognitive system generated by a more developed brain as a result from the immigration experience and from the language exposure. The language exposure would be important to understand how the brain is managing orthographic information from second languages, different from those second languages learned decades ago by ancient immigrant populations.

On the one hand, the genetic differences are not applicable to be investigated concerning this matter [49], on the other hand the cognitive changes could be examined. Rindermann and Thompson [50] had concluded that migrant population presented a growing intelligent quotient (IQ) in the last decades but other data [51] provide some limitations considering the differentiated immigrant groups and their educational attainment in the last years with variability that could impact the IQ in the next decades (see the tendency study of Rindermann and Thompson [50] concerning the cognitive indicators - based in academic scores - of immigrant groups between 1978-2012 and the cognitive evolution predicted for 2060, in the US)

\section{Conclusion and Future Directions}

As future directions for research to test the hypothesis of an evolved cognitive system, caused by immigration constraints (such as the psychological adaptation to the host society) and caused also by the second languages' exposure, based on very recent research advances, we suggest this method: to investigate the new learners (from different immigrant minorities and identified as the more recent immigrant generation or descendants of immigrants' families), in younger ages (differentiating by age groups, properly established by the school grades), with the prerequisite of migratory experience observed in the family (previous generations), and administer them (the new immigrant and second language learners) the same measures administered in the last three decades concerning the verbal reasoning and vocabulary (e.g., the verbal analogy of tests batteries) [52].

On the one hand, this may lead to the confirmation of the hypothesis that populations had already reached new evolution on cognitive mapping (and larger critical period), on the other hand, the psychological adaptation would be a reinforcement and not only a clinical issue for the immigrants' new generations. Also this recommendation for the research with minorities and immigrant school children may develop support for the Rindermand and Pichelmann' study [51] about the cognitive (and IQ) evolution of immigrants in the last decades, but for contexts not so well known, other than North America settings. However, considering the methodological gaps that we still observe for the immigrants' proficiency testing (in several areas and not only for language), the psychological disorders could be enhanced as a main limitation that will constraint the advantage of an extended critical period and cognitive evolving process.

Research community should develop and explore testing procedures and cut-off standards (here specifically we address the Educational Psychology and the Psycholinguistics) for each minority (considering the country of destination). The cut-off may indicate how to place and to respond toward a child immersed in a host school and in a host community, in a proper and differentiated way. As previous literature developed, different minorities, in different countries as receiving countries, are dealing differently with their own inclusion process and, consequently, developing different psychological disorders. In a positive perspective for the research directions, the cognitive advantage could be one of best areas for the Psychology intervention. In a multidisciplinary manner, involving Psychology, Education and Neuropsychology as the major domains.

\section{References}

1. Kim J, Park S, La A, Chang J, Zane N (2016) Counseling services for Asian, Latino/a, and White American students: Initial severity, session attendance, and outcome. Cultur Divers Ethnic Minor Psychol 22: 299-310.

2. Bourque $F$, van der Ven E, Malla A (2011) A meta-analysis of the risk for psychotic disorders among first-and second-generation immigrants. Psychol Med 41: 897-910.

3. Anagnostopoulos C, Triantafyllou K, Xylouris G, Bakatsellos J, Giannakopoulos $\mathrm{G}$ (2016) Migration mental health issues in Europe: the case of Greece. Eur Child Adolesc Psychiatry 25: 119-122.

4. Suárez-Orozco C, Gaytán X, Bang H, Pakes J, O'connor E, et al. (2010) Academic trajectories of newcomer immigrant youth. Dev Psychol 46: 602-618.

5. Guo S, Kataoka S, Bear L, Lau A (2014) Differences in school-based referrals for mental health care: Understanding racial/ethnic disparities between Asian American and Latino youth. Sch Ment Health 6: 27-39.

6. Chow J, Jaffee K, Snowden L (2003) Racial/ethnic disparities in the use of mental health services in poverty areas. Am J Public Health 93: 792-797.

7. Takeuchi D, Zane N, Hong S, Chae D, Gong F, et al. (2007) Immigration-related factors and mental disorders among Asian Americans. Am J Public Health 97: 84-90.

8. Solé-Auro A, Crimmins E (2008) Health of immigrants in European countries. Int Migr Rev 42: 861-876. 
Citation: Figueiredo S, Martins MA, da Silva CF (2018) Psychological Distress of Immigrant Population in Host School Systems and the Critical Period for Second Language Development: A Review. Clin Exp Psychol 4: 192. doi: 10.4172/2471-2701.1000192

Page 5 of 5

9. Sarría-Santamera A, Hijas-Gómez A, Carmona R, Gimeno-Feliú L (2016) Health care usage among immigrants and native-born elderly populations in eleven European countries: results from SHARE. Public Health Rev 37: 28.

10. Crosnoe R, Turley R (2011) K-12 educational outcomes of immigrant youth Future Child 21: 129-152.

11. Lommel LL, Chen JL (2016) The relationship between self-rated health and acculturation in Hispanic and Asian adult immigrants: a systematic review. J Immigr Minor Health 18: 468-478.

12. Figueiredo S, Alves Martins M, Silva C (2016) Second language education context and home language effect: language dissimilarities and variation in immigrant students' outcomes. Int J Multiling 13: 184-212.

13. Figueiredo S, Alves Martins M, Silva C (2016) Modern languages and Modern Learners: different scores among Asian immigrant minorities for academic and language skills development. International Journal of Multidisciplinary Research VI: 71-77.

14. APA (2010) Publication Manual of the American Psychological Association. (6th edn.), American Psychological Association, Washington.

15. Osada N (2001) What Strategy do less proficient learners employ in listening comprehension?: A reappraisal of bottom-up and top-down processing. Journal of Pan-Pacific Association of Applied Linguistics 5: 73-90.

16. Showalter CE, Hayes-Harb R (2013) Unfamiliar orthographic information and second language word learning: A novel lexicon study. Second Language Research 29: 185-200.

17. Barac R, Bialystok E, Castro D, Sanchez M (2014) The cognitive developmen of young dual language learners: A critical review. Early Child Res Q 29: 699714

18. van Staden A, Purcell N (2016) Multi-sensory learning strategies to support spelling development: A case study of second-language learners with auditory processing difficulties. Int J Lang Lit Cult Educ 3: 40-61.

19. Hulstijn J, Young R, Ortega L, Bigelow M, DeKeyser R, et al. (2014) Bridging the gap: Cognitive and social approaches to research in second language learning and teaching. Studies in Second Language Acquisition 36: 361-421.

20. Abebe D, Lien L, Hjelde K (2014) What we know and don't know about mental health problems among immigrants in Norway. J Immigr Minor Health 16: 6067.

21. Kim S, Schwartz S, Perreira K, Juang L (2018) Culture's influence on stressors, parental socialization, and developmental processes in the mental health of children of immigrants. Annu Rev Clin Psychol.

22. Dimitrova R, Chasiotis A, Van de Vijver F (2016) Adjustment outcomes of immigrant children and youth in Europe: A meta-analysis. European Psychologist 21: 150-162.

23. Chateignier C, Dutrévis M, Nugier A, Chekroun $P$ (2009) French-Arab students and verbal intellectual performance:Do they really suffer from a negative intellectual stereotype?. Eur J Dev Psychol 24: 219.

24. Haag N, Heppt B, Roppelt A, Stanat P (2015) Linguistic simplification of mathematics items: effects for language minority students in Germany. Eur $J$ Psychol Educ 30: 145-167.

25. Figueiredo S (2017) Learning Portuguese as a Second Language. Springer, New York.

26. Zhang S, Morris M, Cheng C, Yap A (2013) Heritage-culture images disrup immigrants' second-language processing through triggering first-language interference. Proc Natl Acad Sci U S A 110: 11272-11277.

27. Vandergrift L, Baker S (2015) Learner variables in second language listening comprehension: An exploratory path analysis. Language Learning 65: 390-416.

28. Bassetti B, Escudero P, Hayes-Harb R (2015) Second language phonology a the interface between acoustic and orthographic input. Appl Psycholinguist 36: $1-6$.

29. Kearney L, Draper M, Barón A (2005) Counseling utilization by ethnic minority college students. Cultur Divers Ethnic Minor Psychol 11: 272-285.
30. Abebe D, Lien L, Elstad J (2017) Immigrants' utilization of specialist mental healthcare according to age, country of origin, and migration history: a nationwide register study in Norway. Soc Psychiatry Psychiatr Epidemiol 52: 679-687.

31. Berg J (2009) The level of non-Western immigrants' use of acute psychiatric care compared with ethnic Norwegians over an 8-year period. Nord J Psychiatry 63: 217-222.

32. Yang Y, Wang Q (2016) The relation of emotion knowledge to coping in European American and Chinese immigrant children. J Child Fam Stud 25: 452-463.

33. Levels M, Dronkers J (2008) Educational performance of native and immigrant children from various countries of origin. Ethn Racial Stud 31: 1404-1425.

34. Beiser M, Hou F (2016) Mental health effects of premigration trauma and postmigration discrimination on refugee youth in Canada. J Nerv Ment Dis 204 464-470.

35. Wong C, Schweitzer R (2017) Individual, premigration and postsettlement factors, and academic achievement in adolescents from refugee backgrounds: A systematic review and model. Transcult Psychiatry 54: 756-782.

36. Figueiredo S (2010) Psychological factors and cognitive achievement in linguistic learning [Factores psicológicos e desempenho cognitivo na aprendizagem linguística]. Doctoral thesis, University of Aveiro, Portugal.

37. Johnson JS, Newport EL (1991) Critical period effects on universal properties of language: The status of subjacency in the acquisition of a second language. Cognition 39: 215-258.

38. Lenneberg E, Chomsky N, Marx O (1967) Biological foundations of language. Wiley, New York.

39. Juffs A (2011) Second language acquisition. Wiley Interdiscip Rev Cogn Sci 2: $277-286$.

40. Marinova Todd S, Marshall D, Snow C (2000) Three misconceptions about age and L2 learning. TESOL quarterly 34: 9-34.

41. Mei L, Xue G, Lu Z, He Q, Zhang M, et al. (2013) Orthographic transparency modulates the functional asymmetry in the fusiform cortex: An artificial language training study. Brain Lang 125: 165-172.

42. Vanhove $J$ (2013) The critical period hypothesis in second language acquisition A statistical critique and a reanalysis. PloS one 8: e69172.

43. Scarcella R, Higa C (1982) Input and age differences in second language acquisition. Child-adult differences in second language acquisition.

44. Schumann J (1975) Affective factors and the problem of age in second language acquisition. Language learning 25: 209-235.

45. Verhulp E, Stevens G, Pels T, Van Weert C, Vollebergh W (2017) Lay beliefs about emotional problems and attitudes toward mental health care among parents and adolescents: Exploring the impact of immigration. Cultur Divers Ethnic Minor Psychol 23: 269-280.

46. Sam D (2000) Psychological adaptation of adolescents with immigrant backgrounds. J Soc Psychol 140: 5-25.

47. Weber-Fox C, Neville H (1996) Maturational constraints on functional specializations for language processing: ERP and behavioral evidence in bilingual speakers. J Cogn Neurosci 8: 231-256.

48. Perani D, Abutalebi J (2015) Bilingualism, dementia, cognitive and neura reserve. Curr Opin Neurol 28: 618-625.

49. Hunt E (2012) What makes nations intelligent?. Perspect Psychol Sci 7: 284306.

50. Rindermann H, Thompson J (2016) The cognitive competences of immigrant and native students across the world: An analysis of gaps, possible causes and impact. J Biosoc Sci 48: 66-93.

51. Rindermann H, Pichelmann S (2015) Future cognitive ability: US IQ prediction until 2060 based on NAEP. PloS one 10: e0138412.

52. Alvarado C, Ruef M, Schrank F (2005) Woodcock-Munoz language surveyrevised. Riverside Publishing, Itasca, USA. 